\title{
Electron screening in the liquid-gas mixed phases of nuclear matter
}

\author{
Gentaro Watanabe \\ Department of Physics, University of Tokyo, 7-3-1 Hongo, Bunkyo, Tokyo 113-0033, Japan \\ and Division of Computational Science, The Institute of Physical and \\ Chemical Research (RIKEN), 2-1 Hirosawa, Wako, Saitama 351-0198, Japan
}

\author{
Kei Iida \\ The Institute of Physical and Chemical Research (RIKEN), 2-1 Hirosawa, Wako, Saitama 351-0198, Japan
}

(Dated: October 30, 2018)

\begin{abstract}
Screening effects of electrons on inhomogeneous nuclear matter, which includes spherical, slablike, and rodlike nuclei as well as spherical and rodlike nuclear bubbles, are investigated in view of possible application to cold neutron star matter and supernova matter at subnuclear densities. Using a compressible liquid-drop model incorporating uncertainties in the surface tension, we find that the energy change due to the screening effects broadens the density region in which bubbles and nonspherical nuclei appear in the phase diagram delineating the energetically favorable shape of inhomogeneous nuclear matter. This conclusion is considered to be general since it stems from a model-independent feature that the electron screening acts to decrease the density at which spherical nuclei become unstable against fission and to increase the density at which uniform matter becomes unstable against proton clustering.

PACS numbers: 97.60.Jd, 26.60.+c, 64.75.+g, 21.65.+f
\end{abstract}

\section{INTRODUCTION}

At subnuclear densities and low temperatures where nuclei are so closely packed in a gas consisting mainly of dripped neutrons as to melt into uniform matter, nuclear matter is expected to possess spatially periodic structures composed of rodlike and slablike nuclei and rodlike and spherical bubbles 1]; these nuclei and bubbles are often referred to as nuclear "pasta." Seminal works by Ravenhall et al. 2] and Hashimoto et al. 3] based on liquid-drop models show that a subtle balance between nuclear surface and Coulomb energies determines the energetically most favorable "pasta" shape. Such "pasta"like structures may be encountered in the outer part of a stellar remnant of collapse-driven supernova explosion. In this part, the inhomogeneous nuclear matter would be neutralized and roughly $\beta$-equilibrated by a relativistic degenerate gas of electrons and, during and just after collapse, of electron neutrinos. [Hereafter, such material will be denoted as supernova matter or neutron star matter, according to whether the degenerate neutrino gas coexists or not.] The charge screening action of the electron gas on clumps of protons reduces the Coulomb energy induced by the protons while increasing the kinetic energy of the electron gas. These energy corrections, which have yet to be examined in detail, may affect the density region in which the "pasta" phases are preferred over the low density phase with roughly spherical nuclei and the high density phase of uniform matter.

In this paper we ask the question of how the electron screening changes the energies of zero-temperature supernova matter and neutron star matter at subnuclear densities. For the purpose of answering this question, it is instructive to consider the two opposite limits: perfect screening and no screening. In the case of perfect screening where the electrostatic energy vanishes and the kinetic energy of the electron gas is maximal, a coexisting state of a nucleon liquid of density comparable to the normal nuclear density $n_{0} \simeq 0.16 \mathrm{fm}^{-3}$ and a low density neutron gas can occur at a unique pressure as a phase separated state. On the other hand, in the absence of charge screening, i.e., in the homogeneous limit of the electron gas, the electrostatic energy is maximal while the electron kinetic energy is minimal. In the liquid region in $\beta$ equilibrium, the decrease in the electron Fermi energy as compared to the perfect screening case plays a role in increasing the proton fraction and thus lowering the bulk energy of the nucleon liquid. Due to such decrease in the electron and nucleon bulk energy, a liquid-gas coexisting state can occur for a finite range of pressures as a mixed state composed of alternating liquid-gas regions. At sufficiently low densities, this mixed state is expected to manifest itself as a state in which the liquid regions correspond to roughly spherical nuclei and the gas regions are almost vacant. At densities just below $n_{0}$, as suggested by earlier studies [1], such a mixed state may appear as the "pasta" phases mentioned above, depending on uncertain quantities such as the neutron and proton chemical potentials of a neutron gas and the surface tension in the presence of dripped neutrons. A real situation - partial screening - lies between those two limiting cases, whereas it remains to be clarified how such screening affects the density region of the "pasta" phases through modifications of the bulk and electrostatic energies.

The key quantity of the electron screening is the ratio of the scale of a nucleus or bubble to the Thomas-Fermi 
screening length $\lambda_{\mathrm{TF}}^{(e)}$ as given by

$$
\lambda_{\mathrm{TF}}^{(e)}=\kappa_{e}^{-1}=\left[4 \pi e^{2}\left(\frac{\partial n_{e}^{(0)}}{\partial \mu_{e}^{(0)}}\right)_{n_{e}^{(0)}}\right]^{-1 / 2},
$$

where $n_{e}^{(0)}$ and $\mu_{e}^{(0)}$ are the averaged electron number density and chemical potential, respectively. If the ratio is much smaller than unity, the electron density can be assumed to be everywhere constant to a good approximation. The typical values of $\lambda_{\mathrm{TF}}^{(e)}$ at subnuclear densities for neutron star matter (upper) and supernova matter (lower) can be estimated in the massless limit as

$$
\begin{aligned}
\lambda_{\mathrm{TF}}^{(e)} & =\sqrt{\frac{\pi}{4 \alpha}}\left(k_{e}^{(0)}\right)^{-1} \\
& \simeq \begin{cases}20 \mathrm{fm} & \left(n \simeq 0.5 n_{0}, x \simeq 0.1\right), \\
15 \mathrm{fm} & \left(n \simeq 0.5 n_{0}, x \simeq 0.3\right),\end{cases}
\end{aligned}
$$

where $\alpha$ is the fine structure constant, $k_{e}^{(0)}=\left(3 \pi^{2} n_{e}^{(0)}\right)^{1 / 3}$ is the electron Fermi wave number, and $n$ and $x$ are the nucleon density and proton fraction in the nucleon liquid. These values of $\lambda_{\mathrm{TF}}^{(e)}$ are larger than the typical values of half thickness of a nucleus or bubble, $\sim 5 \mathrm{fm}$, and even half the internuclear spacing, $\sim 10-15 \mathrm{fm}$ (see, e.g., Refs. $[4,[5,6,7,8,[9])$. Consequently, the standard approximation in which no screening is included seems fairly valid. As we shall show within the linearized Thomas-Fermi approximation [10], the screening correction to the density region in which the phases with bubbles and nonspherical nuclei appear is indeed small for the typical values of the surface tension, while the screening makes such a density region larger rather than narrower. This feature will be concluded to be general by investigating how the electron screening affects the conditions for instabilities against fission of spherical nuclei and proton clustering in uniform matter.

Quantum molecular dynamics simulations that have recently been performed by one of the authors successfully reproduce the "pasta" phases expected to occur as the ground state, without imposing any assumptions on nuclear shapes [11]. In this framework, the electron screening has not yet been taken into consideration, whereas proton screening, i.e., Coulombic polarization of the nuclear interior, is automatically incorporated. Before examining the influence of the electron screening on the simulations, it is useful to focus on the qualitative nature of the electron screening effects, which will be clarified in the present work. We remark in passing that the proton screening makes the liquid portion deviate only slightly from uniformity, although the proton screening length is comparable to the scale of the liquid region.

To investigate the electron screening effects on the phase diagram for the ground-state neutron star matter and supernova matter at subnuclear densities, we extend a compressible liquid-drop model of inhomogeneous nuclear matter immersed in the lepton gas as constructed in Refs. [8] and [9] by incorporating the electron density deviation from the non-screening case in the ThomasFermi approximation up to linear order in the electrostatic potential [10]. This model, a generalization of the one developed by Baym, Bethe, and Pethick [12] (hereafter BBP), allows us to obtain the phase diagram in a way dependent on uncertain values of the surface tension. It is essential to take into account such uncertainties; the surface tension is related to the equilibrium size of a nucleus or bubble, which in turn determines the efficiency of the screening.

This paper is composed as follows. In Sec. [II we construct a compressible liquid-drop model for nuclei and bubbles, and write down equilibrium conditions for zerotemperature neutron star matter and supernova matter. Expressions for the electrostatic energy including the electron screening effects are derived in Sec. III] The influence of the electron screening on the phase diagram is discussed in Sec. IV

\section{MODEL OF MATTER AT SUBNUCLEAR DENSITIES}

In this section, we provide the free energy and equilibrium conditions for zero-temperature neutron star matter and supernova matter at densities of order $0.1-1 n_{0}$. The zero-temperature approximation is considered to well describe the energy of matter in the deepest region of neutron star crusts since the temperature of the crusts is typically $\lesssim 10^{9} \mathrm{~K}$, which is much smaller than typical nuclear and electronic excitation energies. For densities considered here, on the other hand, matter under stellar collapse is well characterized by the lepton fraction $Y_{\mathrm{L}}$,

$$
Y_{\mathrm{L}}=\frac{n_{e}^{(0)}+n_{\nu}}{n_{\mathrm{b}}}
$$

where $n_{\nu}$ is the number density of a uniform gas of electron neutrinos, and $n_{\mathrm{b}}$ is the averaged baryon density. The lepton fraction typically amounts to $0.3-0.38$ due to the trapping of electron neutrinos [13, 14]. Hereafter, we set $Y_{\mathrm{L}}=0.3$. Since the degeneracy pressure of the neutrino gas is much larger than the thermal pressure at temperatures of order $10-50 \mathrm{MeV}$, to a first approximation, we shall include the energy of the degenerate neutrino gas into the energy of zero-temperature neutron star matter, and ignore finite temperature effects.

Following Refs. 8] and [9], we consider five phases that consist of spherical nuclei, cylindrical nuclei, planar nuclei, cylindrical bubbles, and spherical bubbles, respectively. Each phase is assumed to be composed of a Coulomb lattice of a single species of nucleus or bubble at a given baryon density $n_{\mathrm{b}}$. We adopt the Wigner-Seitz approximation in evaluating the lattice energy with sufficient accuracy [5]. In this approximation, a cell in the bcc lattice, including a spherical nucleus or bubble of radius $r_{\mathrm{N}}$, is replaced by a Wigner-Seitz cell defined as a 
sphere having radius $r_{\mathrm{c}}$ and the same center. A cylindrical nucleus or bubble having an infinitely long axis and a circular section of radius $r_{\mathrm{N}}$ is taken to be contained in a cylindrical Wigner-Seitz cell having the same axis and a circular section of radius $r_{c}$ in place of a cell in the two-dimensional triangular lattice. For a planar nucleus with thickness $2 r_{\mathrm{N}}$, a Wigner-Seitz cell is identical with a cell in the one-dimensional layered lattice, having width $2 r_{\mathrm{c}}$. The values of $r_{\mathrm{c}}$ for these phases are chosen so that each Wigner-Seitz cell may have zero net charge.

Hereafter we shall concentrate on the charge screening of proton clumps by the electron gas. We shall ignore curvature effects, nucleon pairing effects, shell effects in inhomogeneous nuclear matter [15, 16], and fluctuationinduced displacements of nuclei and bubbles [8, 9], which may have significant consequence to the spatial structure of nuclear matter.

\section{A. Energy of matter}

A compressible liquid-drop model for nuclei and bubbles [1] is useful for investigating the nature of the electron screening. While we assume neutrons and protons to be distributed uniformly inside and outside the nuclei or bubbles and electron neutrinos to be an ideal uniform gas as in earlier investigations, we newly include the screening-induced deviation, $\delta n_{e}(\mathbf{r})$, of the electron number density from the unperturbed constant value $n_{e}^{(0)} ; \delta n_{e}(\mathbf{r})$ will be explicitly calculated within the linear Thomas-Fermi approximation in the next section. Calculating the contributions up to second order in $\delta n_{e}(\mathbf{r})$ accurately, we may write the total energy density, $E_{\text {tot }}$, averaged over a single cell as

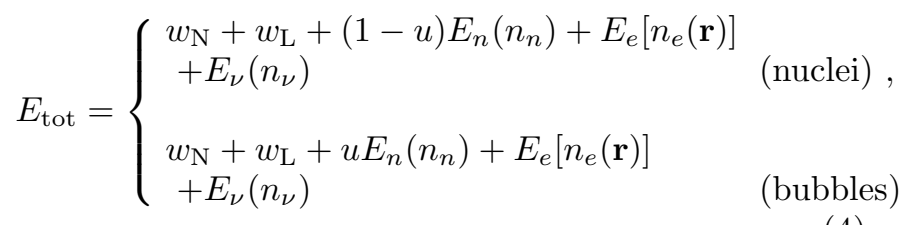

Here $w_{\mathrm{N}}$ is the energy of the nuclear matter region (the region containing protons) in a cell as divided by the cell volume, $w_{\mathrm{L}}$ is the lattice energy per unit volume, $n_{n}$ is the number density of dripped neutrons outside the nuclei or inside the bubbles, $E_{n}, E_{e}$, and $E_{\nu}$ are the energy densities of the neutron matter, of the electron gas, and of the neutrino gas, respectively, and $u$ is the volume fraction occupied by the nuclei or bubbles:

$$
u=\left(\frac{r_{\mathrm{N}}}{r_{c}}\right)^{d}= \begin{cases}\frac{n_{\mathrm{b}}-n_{n}}{n-n_{n}} & \text { (nuclei) }, \\ \frac{n-n_{\mathrm{b}}}{n-n_{n}} & \text { (bubbles) },\end{cases}
$$

where $d$ is the dimensionality defined as $d=1$ for slabs, $d=2$ for cylinders, and $d=3$ for spheres, and $n$ is the nucleon number density inside the nuclear matter region. Note that the contribution of the density deviation $\delta n_{e}(\mathbf{r})$ is included in Eq. (4) through the lattice energy $w_{\mathrm{L}}$ and the electron energy $E_{e}$.

The expressions for $E_{n}, E_{e}$, and $E_{\nu}$ in Eq. (4) are given by

$$
\begin{aligned}
E_{n}\left(n_{n}\right)= & {\left[W\left(k_{n}, 0\right)+m_{n} c^{2}\right] n_{n} } \\
E_{e}\left(n_{e}^{(0)}\right)= & \frac{3}{4} \hbar c k_{e}^{(0)} n_{e}^{(0)} \\
E_{e}\left[n_{e}(\mathbf{r})\right]= & \frac{1}{V_{\mathrm{c}}} \int_{\text {cell }} d^{3} \mathbf{r}\left[E_{e}\left(n_{e}^{(0)}\right)+\frac{\partial E_{e}\left(n_{e}^{(0)}\right)}{\partial n_{e}} \delta n_{e}\right. \\
& \left.+\frac{1}{2} \frac{\partial^{2} E_{e}\left(n_{e}^{(0)}\right)}{\partial n_{e}^{2}} \delta n_{e}^{2}+O\left(\delta n_{e}^{3}\right)\right], \\
\simeq & E_{e}\left(n_{e}^{(0)}\right)\left[1+\frac{2}{9 V_{\mathrm{c}}} \int_{\mathrm{cell}}\left(\frac{\delta n_{e}(\mathbf{r})}{n_{e}^{(0)}}\right)^{2} d^{3} \mathbf{r}\right] \\
E_{\nu}\left(n_{\nu}\right)= & \frac{3}{4} \hbar c k_{\nu} n_{\nu},
\end{aligned}
$$

where $W\left(k_{n}, 0\right)$ with $k_{n}=\left(3 \pi^{2} n_{n} / 2\right)^{1 / 3}$ is the energy per neutron for uniform neutron matter, $V_{\mathrm{c}}$ is the cell volume, and $k_{\nu}=\left(6 \pi^{2} n_{\nu}\right)^{1 / 3}$ is the neutrino Fermi wave number.

For the energy of the nuclear matter region, $w_{\mathrm{N}}$, we adopt a generalized version of the compressible liquiddrop model developed by BBP [12], which gives rise to 8]

$$
\begin{aligned}
& w_{\mathrm{N}}\left(n, x, n_{n}, r_{\mathrm{N}}, r_{\mathrm{c}}, d\right) \\
& =\left\{\begin{array}{c}
u n\left[(1-x) m_{n}+x m_{p}\right] c^{2}+u n W(k, x) \\
\quad+w_{\text {surf }}\left(n, x, n_{n}, r_{\mathrm{N}}, u, d\right)+w_{\mathrm{C}}\left(n, x, r_{\mathrm{N}}, u, d\right) \\
\quad(1-u) n\left[(1-x) m_{n}+x m_{p}\right] c^{2}
\end{array}\right. \\
& +(1-u) n W(k, x)+w_{\text {surf }}\left(n, x, n_{n}, r_{\mathrm{N}}, u, d\right) \\
& +w_{\mathrm{C}}\left(n, x, r_{\mathrm{N}}, u, d\right)
\end{aligned}
$$

- where $m_{n}\left(m_{p}\right)$ is the neutron (proton) rest mass, $W(k, x)$ is the energy per nucleon for uniform nuclear matter of nucleon Fermi wave number $k=\left(3 \pi^{2} n / 2\right)^{1 / 3}$ and proton fraction $x$, as given by BBP [see Eq. (3.19) in Ref. [12]], $w_{\text {surf }}$ is the nuclear surface energy per unit volume, and $w_{\mathrm{C}}$ is the self Coulomb energy (per unit volume) of protons contained in a cell.

This expression for $w_{\mathrm{N}}$ includes three parameters $C_{1}$, $C_{2}$, and $C_{3}$, which are associated with uncertainties in the proton chemical potential $\mu_{p}^{(0)}$ in pure neutron matter as contained in $W(k, x)$ and those in the nuclear surface tension

$$
E_{\text {surf }}=r_{\mathrm{N}} w_{\text {surf }} / u d
$$

The parameter $C_{1}$ determines the magnitude of $\mu_{p}^{(0)}$ (not including the rest mass) as [Eq. (4) in Ref. 8]]

$$
\mu_{p}^{(0)}=-C_{1} n_{n}^{2 / 3}
$$


Hereafter we shall set $C_{1}=400 \mathrm{MeV} \mathrm{fm}^{2}$; this case is consistent with the $n_{n}$ dependence of $\mu_{p}^{(0)}$ obtained from various model calculations as exhibited in Fig. 1 of Ref. 8]. The other two parameters $C_{2}$ and $C_{3}$ are defined as [Eq. (6) in Ref. 8]]

$$
E_{\text {surf }}=C_{2} \tanh \left(\frac{C_{3}}{\mu_{n}^{(0)}}\right) E_{\text {surf }}^{\mathrm{BBP}}
$$

where $\mu_{n}^{(0)}=\partial E_{n} / \partial n_{n}-m_{n} c^{2}$ is the neutron chemical potential in the neutron gas (not including the rest mass), and $E_{\text {surf }}^{\mathrm{BBP}}$ is the BBP-type surface tension [Eq. (7) in Ref. 8]]. As shown in Fig. 1 of Ref. 8] and Fig. 1 of Ref. [9], the surface tension $E_{\text {surf }}$ calculated for $C_{2}=1.0$ and $C_{3}=3.5 \mathrm{MeV}$ agrees well with the Hartree-Fock results obtained by Ravenhall, Bennett, and Pethick [1] using a Skyrme interaction. In the present work, we thus assume $C_{3}=3.5 \mathrm{MeV}$, while we give $C_{2}$ a range of values including unity, 0.1-10. To set such various values for the parameter $C_{2}$ determining the strength of $E_{\text {surf }}$ allows us to investigate the electron screening effects more clearly since the typical size of the nuclear matter region approaches the screening length as $C_{2}$ becomes larger.

\section{B. Equilibrium conditions}

Zero-temperature neutron star matter with nuclei or bubbles of given shape, in its equilibrium, fulfills the conditions for stability of the nuclear matter region against change in the size, neutron drip, $\beta$-decay, and pressurization (see Section 2.2 in Ref. [8]). These conditions arise from minimization of the energy density $E_{\text {tot }}$ with respect to five variables $n, x, n_{n}, r_{\mathrm{N}}$, and $u$ at vanishing $n_{\nu}$ and fixed baryon density $n_{\mathrm{b}}$ given by

$$
n_{\mathrm{b}}= \begin{cases}u n+(1-u) n_{n} & \text { (nuclei) } \\ (1-u) n+u n_{n} & \text { (bubbles) }\end{cases}
$$

as well as under charge neutrality,

$$
n_{e}^{(0)}= \begin{cases}x n u & (\text { nuclei }) \\ x n(1-u) & \text { (bubbles) }\end{cases}
$$

In order to obtain the equilibrium conditions for supernova matter, one has only to repeat the minimization at fixed $Y_{\mathrm{L}}$, Eq. (3), and $n_{\mathrm{b}}$, Eq. (15), rather than at vanishing $n_{\nu}$ and fixed $n_{\mathrm{b}}$.

The expression for the size equilibrium that can be obtained from optimization of $E_{\text {tot }}$ with respect to $r_{\mathrm{N}}$ at fixed $n, x, n_{n}$, and $u$ is

$$
\left.\frac{\partial}{\partial r_{\mathrm{N}}}\left(w_{\mathrm{surf}}+w_{\mathrm{C}+\mathrm{L}}+E_{e}\left[n_{e}(\mathbf{r})\right]\right)\right|_{n, x, n_{n}, u}=0
$$

This expression does not agree with the non-screening formula [see Eq. (14) in Ref. 8]],

$$
w_{\mathrm{surf}}=2 w_{\mathrm{C}+\mathrm{L}}
$$

since the total electrostatic energy density denoted by $w_{\mathrm{C}+\mathrm{L}} \equiv w_{\mathrm{C}}+w_{\mathrm{L}}$ is no longer proportional to $r_{\mathrm{N}}^{2}$ for fixed $u$ (see Sec. III).

The $\beta$-equilibrium condition can be written as

$$
\mu_{e}=\mu_{n}^{(\mathrm{N})}-\mu_{p}^{(\mathrm{N})}+\mu_{\nu}+\left(m_{n}-m_{p}\right) c^{2}
$$

where $\mu_{p}^{(\mathrm{N})}$ is the proton chemical potential in the nuclear matter region given by Eq. (20) in Ref. [8], $\mu_{\nu}=\hbar k_{\nu} c$ is the neutrino chemical potential, which vanishes for neutron star matter, and

$$
\mu_{e}= \begin{cases}\hbar c k_{e}^{(0)}+\left.\frac{2}{9} \frac{1}{n u V_{\mathrm{c}}} \frac{\partial}{\partial x}\left[E_{e}\left(n_{e}^{0}\right) \int\left(\frac{\delta n_{e}(\mathbf{r})}{n_{e}^{(0)}}\right)^{2} d^{3} \mathbf{r}\right]\right|_{n, n_{n}, r_{\mathrm{N}}, r_{\mathrm{c}}} & \text { (nuclei) } \\ \hbar c k_{e}^{(0)}+\left.\frac{2}{9} \frac{1}{n(1-u) V_{\mathrm{c}}} \frac{\partial}{\partial x}\left[E_{e}\left(n_{e}^{0}\right) \int\left(\frac{\delta n_{e}(\mathbf{r})}{n_{e}^{(0)}}\right)^{2} d^{3} \mathbf{r}\right]\right|_{n, n_{n}, r_{\mathrm{N}}, r_{\mathrm{c}}} & \text { (bubbles) }\end{cases}
$$

is the electron chemical potential.

The expressions for the remaining two equilibrium conditions are unchanged from the non-screening case [8, 9]. The condition for the drip equilibrium reads

$$
\mu_{n}^{(\mathrm{N})}=\mu_{n}^{(\mathrm{G})}
$$

where $\mu_{n}^{(\mathrm{N})}$ and $\mu_{n}^{(\mathrm{G})}$ are the neutron chemical potentials in the nuclear matter region and in the neutron gas given by Eqs. (16) and (17) in Ref. [8], respectively. The pressure equilibrium condition can be expressed as

$$
P^{(\mathrm{N})}=P^{(\mathrm{G})}
$$

where $P^{(\mathrm{N})}$ and $P^{(\mathrm{G})}$ are the pressures of the nuclear matter region and of the neutron gas given by Eqs. (22) 
and (23) in Ref. [8], respectively.

In order to see the difference from the non-screening case explicitly, it is instructive to write down the expressions for $\mu_{n}^{(\mathrm{N})}$ and $P^{(\mathrm{N})}$ that can be obtained after expressing $x, r_{\mathrm{N}}$, and $r_{\mathrm{c}}$ in terms of $n_{\mathrm{b}}, n$, and $n_{n}$ through the baryon density (15), the size equilibrium condition (17), and the $\beta$-equilibrium condition (19). The neutron chemical potential $\mu_{n}^{(\mathrm{N})}$ in the nuclear matter region reads

$\mu_{n}^{(\mathrm{N})}=\left\{\begin{array}{l}W(k, x)+\frac{k}{3} \frac{\partial W(k, x)}{\partial k}+\left.\frac{1}{u} \frac{\partial w_{\mathrm{C}+\mathrm{L}}}{\partial n}\right|_{x, r_{\mathrm{N}}, r_{\mathrm{c}}}+\left.\frac{d}{r_{\mathrm{N}}} \frac{\partial E_{\mathrm{surf}}}{\partial n}\right|_{x, n_{n}}+x\left[\mu_{e}-\mu_{\nu}-\left(m_{n}-m_{p}\right) c^{2}\right] \quad \text { (nuclei) }, \\ W(k, x)+\frac{k}{3} \frac{\partial W(k, x)}{\partial k}+\left.\frac{1}{1-u} \frac{\partial w_{\mathrm{C}+\mathrm{L}}}{\partial n}\right|_{x, r_{\mathrm{N}}, r_{\mathrm{c}}}+\left.\frac{d}{r_{\mathrm{N}}} \frac{u}{1-u} \frac{\partial E_{\mathrm{surf}}}{\partial n}\right|_{x, n_{n}}+x\left[\mu_{e}-\mu_{\nu}-\left(m_{n}-m_{p}\right) c^{2}\right] \quad \text { (bubbles) } .\end{array}\right.$

The pressure of the nuclear matter region $P^{(\mathrm{N})}$ reads

$$
P^{(\mathrm{N})}= \begin{cases}\frac{n k}{3} \frac{\partial W(k, x)}{\partial k}-\frac{d-1}{r_{\mathrm{N}}} E_{\mathrm{surf}}+\left.\frac{d n}{r_{\mathrm{N}}} \frac{\partial E_{\mathrm{surf}}}{\partial n}\right|_{x, n_{n}}-\left.\frac{r_{\mathrm{N}}}{d u} \frac{\partial}{\partial r_{\mathrm{N}}}\left(w_{\mathrm{C}+\mathrm{L}}+E_{e}\left[n_{e}(\mathbf{r})\right]\right)\right|_{n u, x, r_{\mathrm{c}}} & \text { (nuclei) } \\ \frac{n k}{3} \frac{\partial W(k, x)}{\partial k}+\frac{d-1}{r_{\mathrm{N}}} E_{\mathrm{surf}}+\left.\frac{d n}{r_{\mathrm{N}}} \frac{u}{1-u} \frac{\partial E_{\mathrm{surf}}}{\partial n}\right|_{x, n_{n}}+\left.\frac{r_{\mathrm{N}}}{d u} \frac{\partial}{\partial r_{\mathrm{N}}}\left(w_{\mathrm{C}+\mathrm{L}}+E_{e}\left[n_{e}(\mathbf{r})\right]\right)\right|_{n(1-u), x, r_{\mathrm{c}}} & \text { (bubbles) . }\end{cases}
$$

For neutron star matter, these expressions are modified from the non-screening expressions, Eqs. (24) and (26) in Ref. [8], due to the difference in the size equilibrium between Eqs. (17) and (18).

Finally, we can calculate the equilibrium values of $n, x, n_{n}, r_{\mathrm{N}}$, and $r_{\mathrm{c}}$ and thus the optimal value of $E_{\mathrm{tot}}$ for neutron star matter (supernova matter) at given $n_{\mathrm{b}}$ and nuclear shape (at $Y_{\mathrm{L}}=0.3$ and at given $n_{\mathrm{b}}$ and nuclear shape) by incorporating Eqs. (23) and (24) into the conditions for drip and pressure equilibria, Eqs. (21) and (22). By comparing the optimal values of $E_{\text {tot }}$ obtained for the five crystalline phases and uniform matter, we can determine the phase giving the smallest energy density at various values of $n_{\mathrm{b}}$ and $C_{2}$ and thereby draw the phase diagram for the ground state neutron star matter and supernova matter, as will be shown in Sec. IV

\section{ELECTROSTATIC ENERGY}

We proceed to derive analytic formulas for the electrostatic energy density $w_{\mathrm{C}+\mathrm{L}}$ in the five crystalline phases by taking into account the electron screening within the framework of the linearized Thomas-Fermi approximation [10]. In this framework, the energy density functional $\varepsilon\left[n_{i}(\mathbf{r}), \phi(\mathbf{r})\right](i=n, p, e, \nu ; \phi$, the electrostatic potential), which is related to the average energy density $E_{\text {tot }}$ as $E_{\text {tot }}=V_{\mathrm{c}}^{-1} \int_{\text {cell }} d^{3} \mathbf{r} \varepsilon\left[n_{i}(\mathbf{r}), \phi(\mathbf{r})\right]$, is expanded up to second order in $\delta n_{e}(\mathbf{r})$. Minimization of the expanded energy density functional with respect to $\phi(\mathbf{r})$ and $\delta n_{e}(\mathbf{r})$ leads to the Poisson equation,

$$
\nabla^{2} \phi(\mathbf{r})-\kappa_{e}^{2} \phi(\mathbf{r})
$$

$$
\begin{aligned}
& =-4 \pi n_{Q}(\mathbf{r}) \\
& = \begin{cases}-4 \pi e\left[n x \theta\left(r_{\mathrm{N}}-|\mathbf{r}|\right)-n_{e}^{(0)}\right] & \text { (nuclei) } \\
-4 \pi e\left[n x \theta\left(|\mathbf{r}|-r_{\mathrm{N}}\right)-n_{e}^{(0)}\right] & \text { (bubbles) }\end{cases}
\end{aligned}
$$

where $n_{Q}$ is the local charge density in the non-screening limit, and the relation between $\delta n_{e}(\mathbf{r})$ and $\phi$,

$$
\delta n_{e}(\mathbf{r})=\frac{\kappa_{e}^{2}}{4 \pi e^{2}} e \phi(\mathbf{r}) .
$$

For planar configuration, we take Cartesian coordinates in which the $z$-axis is normal to the nuclear surface and the origin is located on the central plane of the nucleus; for cylindrical configuration, cylindrical coordinates in which the radial coordinate $\rho$ is normal to the surface of the nucleus or bubble and the line of $\rho=0$ coincides with the symmetry axis of the nucleus or bubble; for spherical configuration, spherical coordinates in which the radial coordinate $r$ is normal to the surface of the nucleus or bubble and the origin is located at its center. In physical realizations, the electrostatic potential and its derivative must be continuous throughout the system. Thus, appropriate boundary conditions are that the derivative of the electrostatic potential at the origin and the cell boundary is zero, i.e., $\phi^{\prime}(0)=0$ and $\phi^{\prime}\left(r_{\mathrm{c}}\right)=0$; and that the electrostatic potential and its derivative are continuous at the surface of the nucleus or bubble.

We turn to the solutions to the Poisson equation (25) for slablike nuclei, cylindrical nuclei and bubbles, and spherical nuclei and bubbles. The results for the electrostatic potential read 
- slab

$$
\phi(z)= \begin{cases} \pm \frac{4 \pi n x e}{\kappa_{e}^{2}}\left[-\frac{\sinh \left[\kappa_{e}\left(r_{\mathrm{c}}-r_{\mathrm{N}}\right)\right]}{\sinh \left(\kappa_{e} r_{\mathrm{c}}\right)} \cosh \left(\kappa_{e}|z|\right)+(1-u)\right] & 0 \leq|z| \leq r_{\mathrm{N}} \\ \pm \frac{4 \pi n x e}{\kappa_{e}^{2}}\left[\frac{\sinh \left(\kappa_{e} r_{\mathrm{N}}\right)}{\sinh \left(\kappa_{e} r_{\mathrm{c}}\right)} \cosh \left[\kappa_{e}\left(r_{\mathrm{c}}-|z|\right)\right]-u\right] & r_{\mathrm{c}} \geq|z|>r_{\mathrm{N}}\end{cases}
$$

- cylinder

$$
\phi(\rho)= \begin{cases} \pm \frac{4 \pi n x e}{\kappa_{e}^{2}}\left[\frac{K_{1}\left(\kappa_{e} r_{\mathrm{c}}\right) I_{1}\left(\kappa_{e} r_{\mathrm{N}}\right)-I_{1}\left(\kappa_{e} r_{\mathrm{c}}\right) K_{1}\left(\kappa_{e} r_{\mathrm{N}}\right)}{I_{1}\left(\kappa_{e} r_{\mathrm{c}}\right)} \kappa_{e} r_{\mathrm{N}} I_{0}\left(\kappa_{e} \rho\right)+(1-u)\right] & 0 \leq \rho \leq r_{\mathrm{N}}, \\ \pm \frac{4 \pi n x e}{\kappa_{e}^{2}}\left[\kappa_{e} r_{\mathrm{N}} I_{1}\left(\kappa_{e} r_{\mathrm{N}}\right)\left\{\frac{K_{1}\left(\kappa_{e} r_{\mathrm{c}}\right)}{I_{1}\left(\kappa_{e} r_{\mathrm{c}}\right)} I_{0}\left(\kappa_{e} \rho\right)+K_{0}\left(\kappa_{e} \rho\right)\right\}-u\right] & r_{\mathrm{c}} \geq \rho>r_{\mathrm{N}},\end{cases}
$$

- sphere

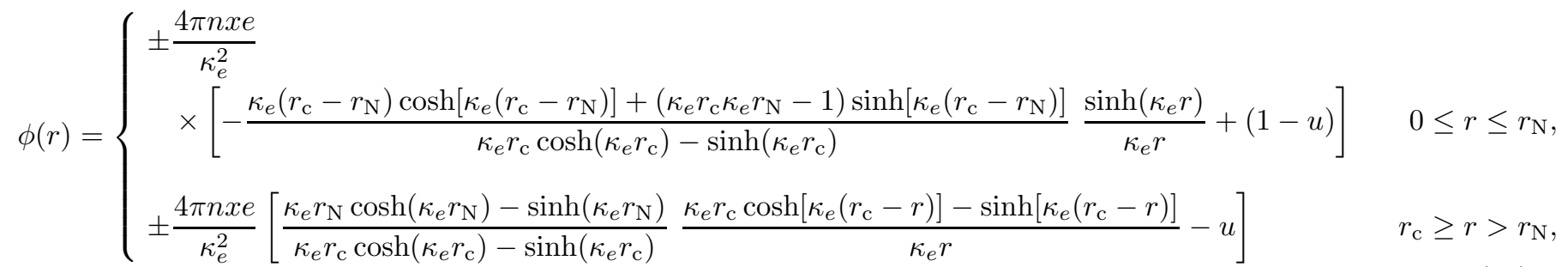

where the upper plus (lower minus) sign corresponds to nuclei (bubbles); $I_{n}$ and $K_{n}$ are the $n$-th modified Bessel functions.
Correspondingly, the electric field $E$ can be obtained from the derivative of the potential $\phi$ as

- slab

$$
E(z)= \begin{cases} \pm \frac{4 \pi n x e}{\kappa_{e}} \frac{\sinh \left[\kappa_{e}\left(r_{\mathrm{c}}-r_{\mathrm{N}}\right)\right]}{\sinh \left(\kappa_{e} r_{\mathrm{c}}\right)} \sinh \left(\kappa_{e}|z|\right) \frac{z}{|z|} & 0 \leq|z| \leq r_{\mathrm{N}} \\ \pm \frac{4 \pi n x e}{\kappa_{e}} \frac{\sinh \left(\kappa_{e} r_{\mathrm{N}}\right)}{\sinh \left(\kappa_{e} r_{\mathrm{c}}\right)} \sinh \left[\kappa_{e}\left(r_{\mathrm{c}}-|z|\right)\right] \frac{z}{|z|} & r_{\mathrm{c}} \geq|z|>r_{\mathrm{N}}\end{cases}
$$

- cylinder

$$
E(\rho)= \begin{cases} \pm \frac{4 \pi n x e}{\kappa_{e}} \frac{I_{1}\left(\kappa_{e} r_{\mathrm{c}}\right) K_{1}\left(\kappa_{e} r_{\mathrm{N}}\right)-K_{1}\left(\kappa_{e} r_{\mathrm{c}}\right) I_{1}\left(\kappa_{e} r_{\mathrm{N}}\right)}{I_{1}\left(\kappa_{e} r_{\mathrm{c}}\right)} \kappa_{e} r_{\mathrm{N}} I_{1}\left(\kappa_{e} \rho\right) & 0 \leq \rho \leq r_{\mathrm{N}} \\ \pm \frac{4 \pi n x e}{\kappa_{e}} \kappa_{e} r_{\mathrm{N}} I_{1}\left(\kappa_{e} r_{\mathrm{N}}\right)\left[K_{1}\left(\kappa_{e} \rho\right)-\frac{K_{1}\left(\kappa_{e} r_{\mathrm{c}}\right)}{I_{1}\left(\kappa_{e} r_{\mathrm{c}}\right)} I_{1}\left(\kappa_{e} \rho\right)\right] & r_{\mathrm{c}} \geq \rho>r_{\mathrm{N}}\end{cases}
$$

- sphere

$$
E(r)= \begin{cases} \pm \frac{4 \pi n x e}{\kappa_{e}} \frac{\kappa_{e}\left(r_{\mathrm{c}}-r_{\mathrm{N}}\right) \cosh \left[\kappa_{e}\left(r_{\mathrm{c}}-r_{\mathrm{N}}\right)\right]+\left(\kappa_{e} r_{\mathrm{c}} \kappa_{e} r_{\mathrm{N}}-1\right) \sinh \left[\kappa_{e}\left(r_{\mathrm{c}}-r_{\mathrm{N}}\right)\right]}{\kappa_{e} r_{\mathrm{c}} \cosh \left(\kappa_{e} r_{\mathrm{c}}\right)-\sinh \left(\kappa_{e} r_{\mathrm{c}}\right)} & \\ \times \frac{\kappa_{e} r \cosh \left(\kappa_{e} r\right)-\sinh \left(\kappa_{e} r\right)}{\left(\kappa_{e} r\right)^{2}} & 0 \leq r \leq r_{\mathrm{N}} \\ \pm \frac{4 \pi n x e}{\kappa_{e}} \frac{\kappa_{e} r_{\mathrm{N}} \cosh \left(\kappa_{e} r_{\mathrm{N}}\right)-\sinh \left(\kappa_{e} r_{\mathrm{N}}\right)}{\kappa_{e} r_{\mathrm{c}} \cosh \left(\kappa_{e} r_{\mathrm{c}}\right)-\sinh \left(\kappa_{e} r_{\mathrm{c}}\right)} & \\ \times \frac{\kappa_{e}\left(r_{\mathrm{c}}-r\right) \cosh \left[\kappa_{e}\left(r_{\mathrm{c}}-r\right)\right]+\left(\kappa_{e} r_{\mathrm{c}} \kappa_{e} r-1\right) \sinh \left[\kappa_{e}\left(r_{\mathrm{c}}-r\right)\right]}{\left(\kappa_{e} r\right)^{2}} & r_{\mathrm{c}} \geq r>r_{\mathrm{N}}\end{cases}
$$


where the upper plus (lower minus) sign corresponds to nuclei (bubbles) as in Eqs. (27)-(29).

We remark that the electron density deviation $\delta n_{e}$, which can be obtained by substituting the solutions for $\phi$ given by Eqs. (27) - 29) into Eq. (26) does not violate charge neutrality in a cell. This is because $\int_{\text {cell }} \delta n_{e} d^{3} \mathbf{r} \propto$ $\int_{\text {cell }} \phi d^{3} \mathbf{r}=0$.

The total electrostatic energy $W_{\mathrm{C}+\mathrm{L}}$ in a cell is given by

$$
W_{\mathrm{C}+\mathrm{L}}=\frac{1}{8 \pi} \int_{\text {cell }} E^{2} d^{3} \mathbf{r} .
$$

The electrostatic energy density $w_{\mathrm{C}+\mathrm{L}}$ can thus be calculated by substituting Eqs. (30)- (32) into Eq. (33) as

$$
w_{\mathrm{C}+\mathrm{L}}=2 \pi(n x e)^{2} r_{\mathrm{N}}^{2} u f_{d}^{(\mathrm{screen})}\left(\kappa_{e} r_{\mathrm{c}}, \kappa_{e} r_{\mathrm{N}}\right)
$$

with

- slab

$$
f_{1}^{(\text {screen })}(s, t)=\frac{1}{2 t^{2}}\left\{-\frac{s}{t} \frac{\sinh ^{2} t}{\sinh ^{2} s}+\frac{1}{t} \frac{\sinh t}{\sinh s} \sinh (s-t)+2 \frac{\sinh t}{\sinh s} \cosh (s-t)-1\right\}
$$

- cylinder

$$
\begin{aligned}
& f_{2}^{\text {(screen })}(s, t)=I_{1}^{2}(t)\left[\frac{K_{1}(t)}{I_{1}(t)}\left\{\frac{I_{1}(t)}{K_{1}(t)} K_{0}(t) K_{2}(t)-\frac{K_{1}(t)}{I_{1}(t)} I_{0}(t) I_{2}(t)-2 \frac{K_{1}(s)}{I_{1}(s)}\left(I_{1}^{2}(t)-I_{0}(t) I_{2}(t)\right)\right\}\right. \\
& +\left(\frac{s}{t}\right)^{2} K_{1}^{2}(s)\left\{2-\frac{I_{0}(s) I_{2}(s)}{I_{1}^{2}(s)}-\frac{K_{0}(s) K_{2}(s)}{K_{1}^{2}(s)}\right\}
\end{aligned}
$$

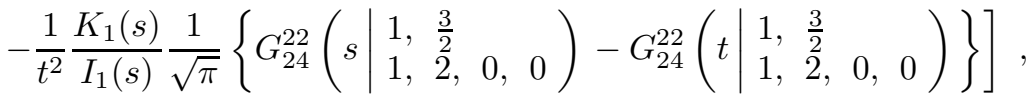

- sphere

$$
\begin{aligned}
f_{3}^{(\text {screen })}(s, t)=\frac{3}{2 t^{6}} & \frac{1}{(s \cosh s-\sinh s)^{2}} \\
\times & \times\left[\left\{-\left(1+(s-t)\left(s^{2} t-s-t\right)\right)+(1+s(s-t)) \cosh [2(s-t)]\right.\right. \\
& \left.\quad+\frac{1}{2}\left(s^{2} t-4 s+t\right) \sinh [2(s-t)]\right\}(t \cosh t-\sinh t)^{2} \\
& \left.\quad+\{(s-t) \cosh (s-t)+(s t-1) \sinh (s-t)\}^{2}\left(t^{2}+t \cosh t \sinh t-2 \sinh ^{2} t\right)\right],
\end{aligned}
$$

where $G_{24}^{22}$ is the Meijer's G-function defined as

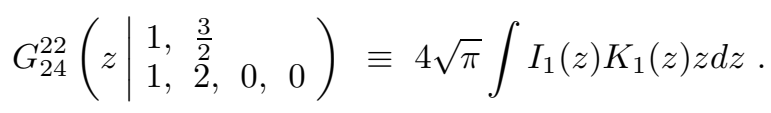

In Figs. 1] and 2 we plot the ratio $f_{d}^{(\text {screen })}\left(\kappa_{e} r_{\mathrm{c}}, \kappa_{e} r_{\mathrm{N}}\right) / f_{d}(u)$, where $f_{d}(u)$ is the nonscreening limit given by

$$
f_{d}(u)=\frac{1}{d+2}\left[\frac{2}{d-2}\left(1-\frac{d u^{1-2 / d}}{2}\right)+u\right] .
$$

This expression can be obtained by taking $\kappa_{e} \rightarrow 0$ in Eqs. (35) - (37). We find from Fig. 11 that as $\kappa_{e}$ increases (i.e., the electron density $n_{e}^{(0)}$ increases), the ratio $f_{d}^{(\text {screen })} / f_{d}$ decreases most rapidly for the slablike configuration while most slowly for the spherical configuration. This configuration dependence holds also for increase in $r_{\mathrm{N}}$ and $r_{\mathrm{c}}$, as can be seen in Fig. 2] We may thus conclude that for fixed values of $r_{\mathrm{N}}, r_{\mathrm{c}}$, and $n_{e}^{(0)}$, the screening-induced reduction in the electrostatic energy is larger for the structure of lower dimensionality.

In Figs. 3 and 4 we illustrate the charge distributions for the five crystalline phases in neutron star matter and supernova matter, respectively. We can see from these figures that in both cases the magnitude of the electron density deviation $\left|\delta n_{e}\right|$ induced by the screening is much smaller than that of the unperturbed charge number density $\left|n_{Q}\right| / e$. This ensures the validity of the linearized Thomas-Fermi approximation adopted here. 


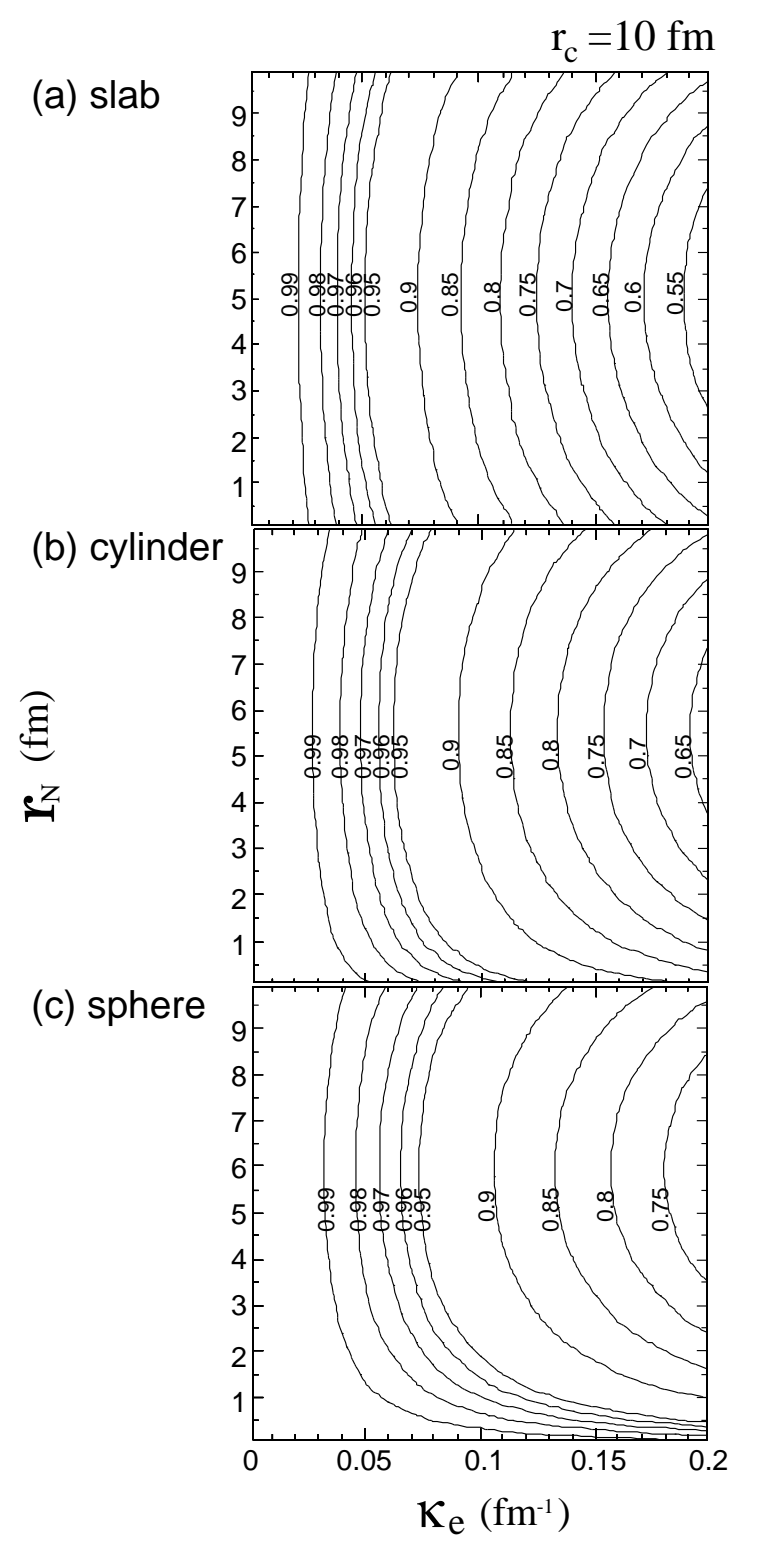

FIG. 1: Contour plot of the ratio $f_{d}^{\text {(screen) }} / f_{d}$ on the $\kappa_{e}$ versus $r_{\mathrm{N}}$ plane for (a) slablike nuclei $(d=1)$, (b) cylindrical nuclei or bubbles $(d=2)$, and (c) spherical nuclei or bubbles $(d=3)$. The Wigner-Seitz cell radius $r_{\mathrm{c}}$ is fixed at $r_{\mathrm{c}}=10 \mathrm{fm}$. In the Coulomb limit of $\kappa_{e} \rightarrow 0$, the ratio reduces to unity.

\section{SCREENING CORRECTIONS}

We now examine the influence of the electron screening on the phase diagrams of the ground-state neutron star matter and supernova matter at subnuclear densities. In Figs. 5 and 6 we draw the phase diagrams of neutron star matter and supernova matter, respectively, on the $n_{\mathrm{b}}$ versus $C_{2}$ plane for the cases with and without screening. As can be seen from these figures, the screening leads to slight expansion of the density region in which the "pasta" phases appear, and this expansion is larger in

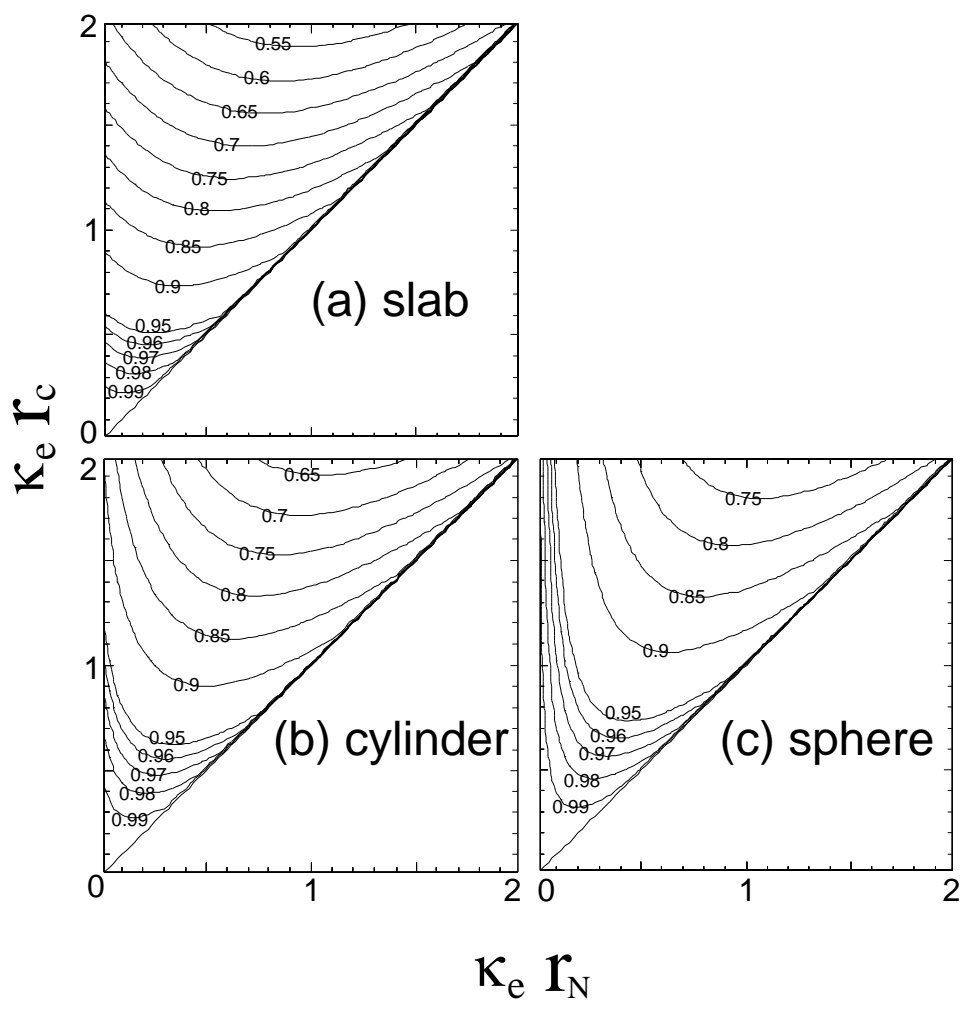

FIG. 2: Contour plot of the ratio $f_{d}^{\text {(screen) }} / f_{d}$ on the $\kappa_{e} r_{\mathrm{N}}$ versus $\kappa_{e} r_{\mathrm{c}}$ plane for (a) slablike nuclei $(d=1)$, (b) cylindrical nuclei or bubbles $(d=2)$, and (c) spherical nuclei or bubbles $(d=3)$.

supernova matter than in neutron star matter.

In order to consider why the screening is more effective in supernova matter than in neutron star matter, it is useful to plot the screening length $\lambda_{\mathrm{TF}}^{(e)}$ and the spatial scales, $r_{\mathrm{N}}$ and $r_{\mathrm{c}}$, as in Figs. 7 and 8 By comparing these figures, we can see that the ratios $r_{\mathrm{N}} / \lambda_{\mathrm{TF}}^{(e)}$ and $r_{\mathrm{c}} / \lambda_{\mathrm{TF}}^{(e)}$, whose increase makes the electron screening more effective, is larger in supernova matter than in neutron star matter. This is because of smaller $\lambda_{\mathrm{TF}}^{(e)}$ in supernova matter.

We also see from Figs. 17 and 8 that the electron screening acts to increase $r_{\mathrm{N}}$ and $r_{\mathrm{c}}$ by a small amount, while keeping the ratio $r_{\mathrm{N}} / r_{\mathrm{c}}$, or equivalently, $u$, almost unchanged. Such increase in $r_{\mathrm{N}}$ stems from the fact that as discussed in Sec. III the electron screening modifies the condition for size equilibrium from Eq. (18) to Eq. (17), which can be rewritten as

$$
w_{\text {surf }}=2\left(w_{\mathrm{C}+\mathrm{L}, 0}+\delta w_{\mathrm{C}+\mathrm{L}}+\delta E_{e}\right) .
$$

Here, $w_{\mathrm{C}+\mathrm{L}, 0}=2 \pi(n x e)^{2} r_{\mathrm{N}}^{2} u f_{d}(u)$ is the total electrostatic energy density in the non-screening limit, and $\delta w_{\mathrm{C}+\mathrm{L}}$ and $\delta E_{e}$ are the corrections to Eq. (18) due to the screening-induced changes in the electrostatic energy and the electron energy, respectively, which are proportional to $\kappa_{e}^{2}$ in leading order. Up to $O\left(\kappa_{e}^{2}\right)$, the sum $\delta w_{\mathrm{C}+\mathrm{L}}$ and 


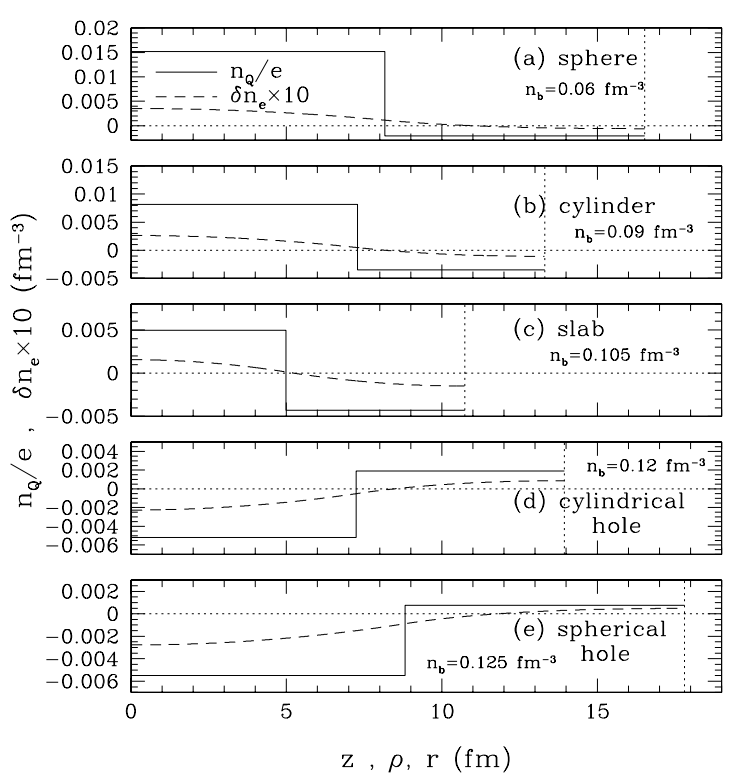

FIG. 3: Charge distribution in a Wigner-Seitz cell in neutron star matter for the phases with (a) spherical nuclei, (b) cylindrical nuclei, (c) slablike nuclei, (d) cylindrical bubbles, and (e) spherical bubbles, calculated at typical baryon densities for $C_{2}=1.0$. The solid lines denote the unperturbed local charge density $n_{Q}(\mathbf{r})$, divided by $e$, and the dashed lines denote the screening-induced deviation $\delta n_{e}(\mathbf{r})$ of the electron number density, multiplied by ten. The vertical dotted lines are the cell boundaries.

$\delta E_{e}$ is negative since $\delta w_{\mathrm{C}+\mathrm{L}}$, which is negative, is more important than $\delta E_{e}$, which is positive. Consequently, the screening makes the equilibrium value of $r_{\mathrm{N}}$ larger than that in the non-screening limit, given by

$$
r_{\mathrm{N}}^{(0)}=\left[\frac{d E_{\mathrm{surf}}}{4 \pi(n x e)^{2} f_{d}}\right]^{1 / 3} .
$$

On the other hand, the negligible screening effect on $u$ suggests that the pressure corrections due to the screening through the Coulomb pressure and the electron pressure [see the last two terms in the right side of Eq. (24)] are negligibly small.

We proceed to see how the phase structure changes with the strength of the surface tension, $C_{2}$, and the baryon density $n_{\mathrm{b}}$. We find from Figs. 5 and 6 that as $C_{2}$ decreases, the phase boundaries in the case with screening approach those in the case without screening. This is consistent with the fact that for weaker surface tension, the equilibrium size of the spatial structure becomes smaller, leading to smaller $r_{\mathrm{N}} / \lambda_{\mathrm{TF}}^{(e)}$ and $r_{\mathrm{c}} / \lambda_{\mathrm{TF}}^{(e)}$. As $n_{\mathrm{b}}$ increases with $C_{2}$ fixed, on the other hand, the screening induced change in the phase boundaries becomes more appreciable; the increase in the transition density between the cylindrical hole to the spherical hole phase is larger than that between the slab to the cylindrical hole phase while being smaller than that between the

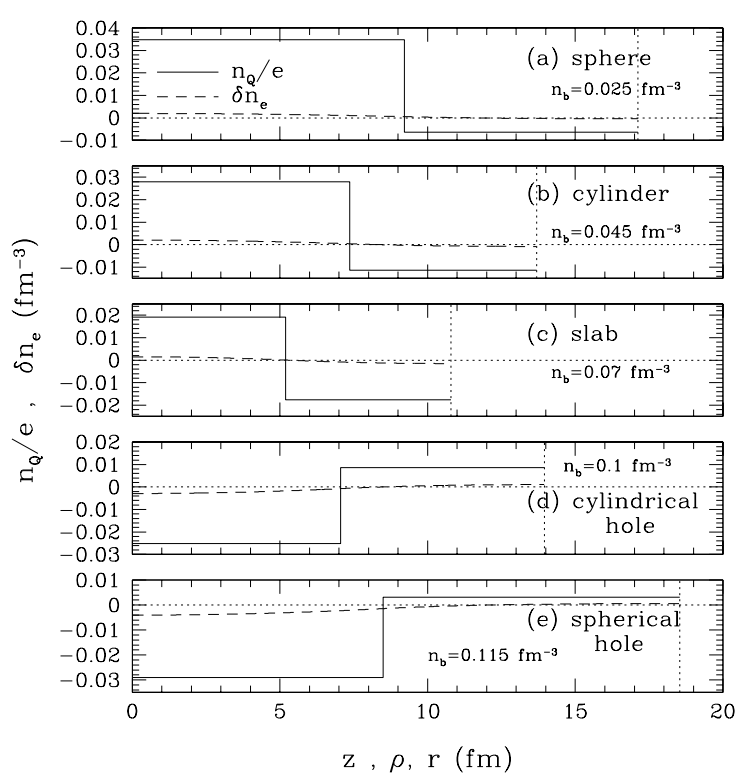

FIG. 4: Charge distribution in a Wigner-Seitz cell in supernova matter for the phases with (a) spherical nuclei, (b) cylindrical nuclei, (c) slablike nuclei, (d) cylindrical bubbles, and (e) spherical bubbles, calculated at typical baryon densities for $C_{2}=1.0$ and $Y_{\mathrm{L}}=0.3$. The solid lines denote the unperturbed local charge density $n_{Q}(\mathbf{r})$, divided by $e$, and the dashed lines denote the screening-induced deviation $\delta n_{e}(\mathbf{r})$ of the electron number density. The vertical dotted lines are the cell boundaries.

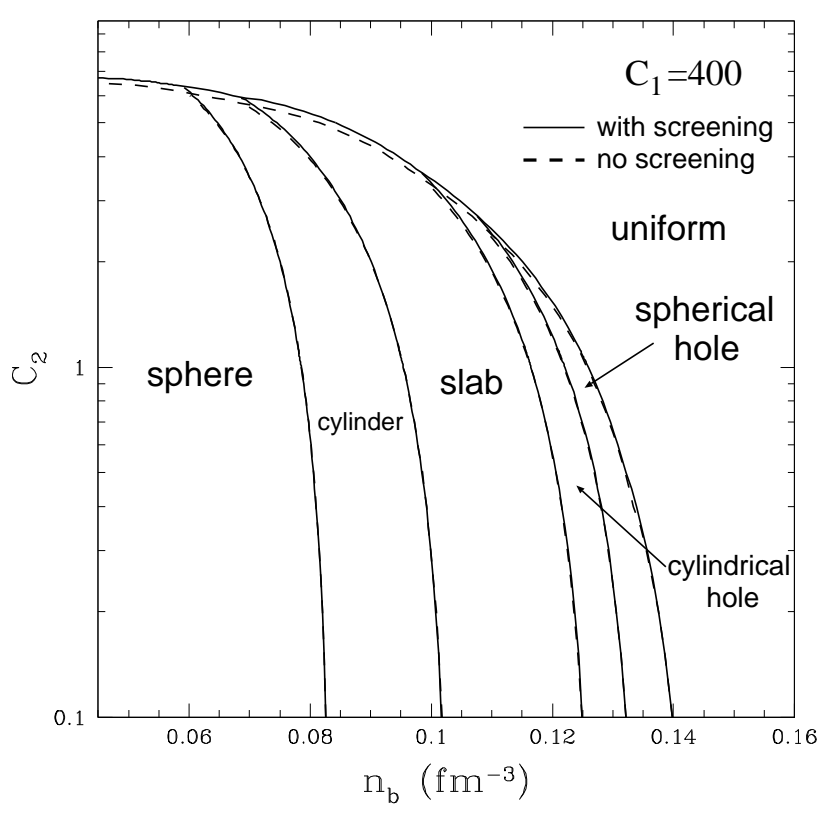

FIG. 5: Zero-temperature phase diagram of neutron star matter on the $n_{\mathrm{b}}$ versus $C_{2}$ plane. The solid lines are the phase boundaries obtained for the case allowing for the electron screening. The dashed lines are for the case ignoring the electron screening, which are taken from the lower left panel in Fig. 3 in Ref. [8]. 


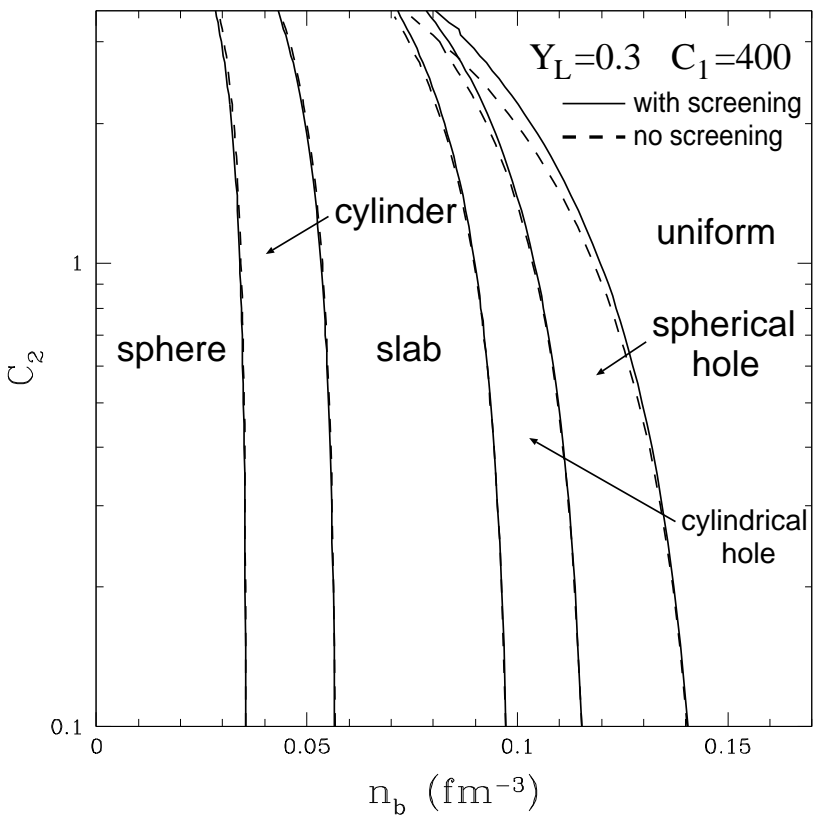

FIG. 6: Zero-temperature phase diagram of supernova matter on the $n_{\mathrm{b}}$ versus $C_{2}$ plane. The solid lines are the phase boundaries obtained for the case allowing for the electron screening. The dashed lines are for the case ignoring the electron screening, which are taken from the lower left panel in Fig. 3 in Ref. 9].

spherical hole to the uniform phase. This is partly because $\lambda_{\mathrm{TF}}^{(e)}$ decreases with increasing density and partly because at fixed $n_{\mathrm{b}}$, the higher dimensionality has the larger equilibrium values of $r_{\mathrm{N}}$ and $r_{\mathrm{c}}$. This dimensionality dependence, which was also obtained in earlier investigations based on various nuclear models (see, e.g., Refs. [4, 5, 8, 9]), stems from the fact that generally the equilibrium values of the surface energy density $w_{\text {surf }}$ and surface tension $E_{\text {surf }}$ are almost degenerate among the five crystalline phases at fixed $n_{\mathrm{b}}$ and thus the equilibrium value of $r_{\mathrm{N}}$ behaves roughly as $r_{\mathrm{N}} \propto d$ [see Eq. (12)].

In order to examine the influence of the screening on the phase boundaries in further detail, we list in Table 【 the transition densities calculated for neutron star matter and supernova matter at a typical value of $C_{2}=1.0$. We thus find that the "pasta" phases, as a whole, are slightly enlarged by the electron screening in such a way that the phase boundaries associated with the bubble phases move into a higher density, whereas the others move into a lower density. Such movement stems from the dimensionality dependence of the quantity, $f_{d}^{\text {(screen) }} / f_{d}$, characterizing the screening correction to the electrostatic energy: At fixed $r_{\mathrm{N}}, r_{\mathrm{c}}$, and $n_{e}^{(0)}$, as was found from Figs. [1] and 2] $f_{d}^{\text {(screen) }} / f_{d}$ is smaller and hence the screening is more efficient for lower dimensionality. We remark in passing that the screening correction to the electrostatic energy dominates over the screening correction to the

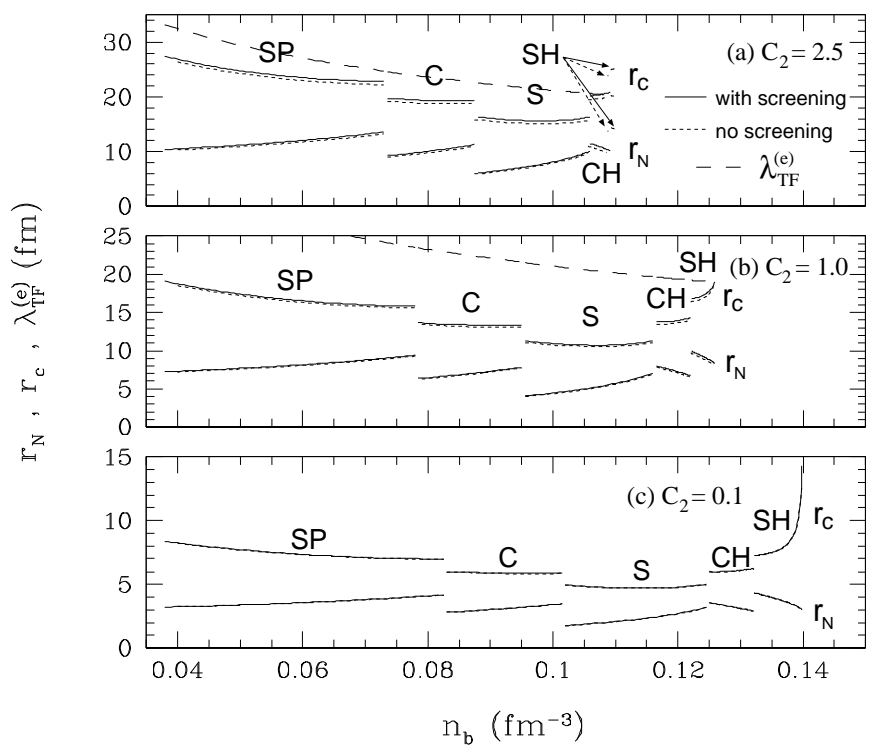

FIG. 7: Size of a nucleus or bubble, $r_{\mathrm{N}}$, and of a WignerSeitz cell, $r_{\mathrm{c}}$, in neutron star matter calculated for $C_{2}=0.1$, 1.0, and 2.5. The Thomas-Fermi screening length $\lambda_{\mathrm{TF}}^{(e)}$ is also plotted. The solid lines are the results for the case with screening, and the dashed lines are the results for the case without screening, which are taken from Fig. 5 in Ref. [8]. The symbols SP, C, S, CH, and SH stand for sphere, cylinder, slab, cylindrical hole, and spherical hole, respectively.

TABLE I: Transition densities (in $\mathrm{fm}^{-3}$ ) in neutron star matter (NSM) and supernova matter (SNM), calculated at $C_{2}=1.0$. The lepton fraction $Y_{\mathrm{L}}$ is set at 0.3 for supernova matter. The symbols $\bigcirc$ and $\times$ denote the cases with and without screening, respectively. The symbols SP, C, S, CH, $\mathrm{SH}$, and $\mathrm{U}$ stand for sphere, cylinder, slab, cylindrical hole, spherical hole, and uniform matter, respectively.

\begin{tabular}{cccccccc}
\hline & screening & $\mathrm{SP} \leftrightarrow \mathrm{C}$ & $\mathrm{C} \leftrightarrow \mathrm{S}$ & $\mathrm{S} \leftrightarrow \mathrm{CH}$ & $\mathrm{CH} \leftrightarrow \mathrm{SH}$ & $\mathrm{SH} \leftrightarrow \mathrm{U}$ \\
$\mathrm{NSM}$ & $\times$ & 0.07854 & 0.09537 & 0.11636 & 0.12191 & 0.12571 \\
$\mathrm{NSM}$ & $\bigcirc$ & 0.07849 & 0.09535 & 0.11648 & 0.12206 & 0.12592 \\
$\mathrm{SNM}$ & $\times$ & 0.03433 & 0.05364 & 0.08965 & 0.10364 & 0.11812 \\
$\mathrm{SNM}$ & $\bigcirc$ & 0.03407 & 0.05334 & 0.08990 & 0.10410 & 0.11974 \\
\hline \hline
\end{tabular}

electron energy.

The decrease in the transition density between the phases with spherical and with cylindrical nuclei can be understood from the condition for fission instability of spherical nuclei in a Coulomb lattice. Up to $O\left(u^{1 / 3}\right)$, this condition reads [18]

$$
w_{\mathrm{C}} \geq 2 w_{\text {surf }}
$$

where $w_{\mathrm{C}}=4 \pi(n x e)^{2} u r_{\mathrm{N}}^{2} / 5$ is the Coulomb self energy of the nucleus, divided by the cell volume. Condition 


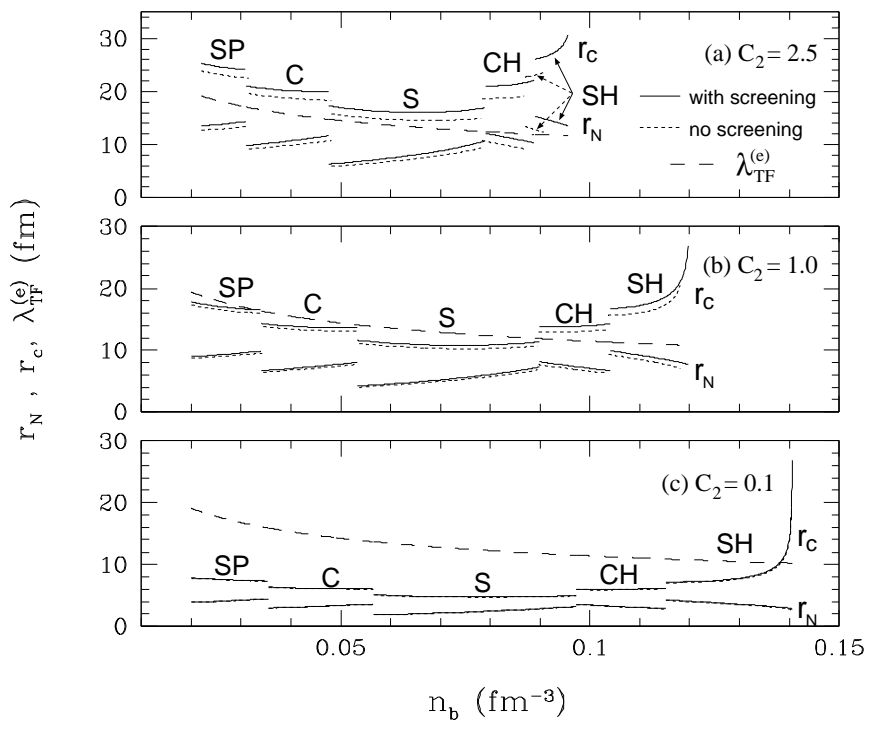

FIG. 8: Size of a nucleus or bubble, $r_{\mathrm{N}}$, and of a WignerSeitz cell, $r_{\mathrm{c}}$, in supernova matter calculated for $Y_{\mathrm{L}}=0.3$ and $C_{2}=0.1,1.0$, and 2.5. The Thomas-Fermi screening length $\lambda_{\mathrm{TF}}^{(e)}$ is also plotted. The solid lines are the results for the case with screening, and the dashed lines are the results for the case without screening, which are taken from Fig. 5 in Ref. 9]. The symbols SP, C, S, CH, and SH stand for sphere, cylinder, slab, cylindrical hole, and spherical hole, respectively.

(42) provides a critical size,

$$
r_{\mathrm{N}}^{\mathrm{crit}}=\left[\frac{30 E_{\mathrm{surf}}}{4 \pi(n x e)^{2}}\right]^{1 / 3}
$$

In the absence of screening, the equilibrium nuclear size $r_{\mathrm{N}}^{(0)}$, given by Eq. (41) in which $f_{d=3}$ is retained up to $O\left(u^{1 / 3}\right)$, reaches this critical size when $u=1 / 8$. In the presence of screening, as in Figs. 7 and 8 the equilibrium value of $r_{\mathrm{N}}$ is larger than $r_{\mathrm{N}}^{(0)}$ at given $n_{\mathrm{b}}$. Since the equilibrium value of $r_{\mathrm{N}}$ increases with $n_{\mathrm{b}}$ for spherical nuclei, the density region in which the spherical nuclei are unstable against fission extends to a lower density.

The increase in the density at which the system turns into uniform matter is the most salient feature induced by the screening; as can be seen from Table【 the density increase for typical values of the surface tension $\left(C_{2} \simeq 1\right)$ amounts to $\sim 2 \times 10^{-4} \mathrm{fm}^{-3}$ for neutron star matter and $\sim 0.0015 \mathrm{fm}^{-3}$ for supernova matter. This feature can be understood from the condition for proton clustering instability in $\beta$-equilibrated uniform nuclear matter, which was originally obtained by BBP 12] for neutron star matter. (For supernova matter, one can follow the same argument as that for neutron star matter since the presence of trapped neutrinos makes no difference in the argument.) They expanded the energy density functional $E\left[n_{i}(\mathbf{r})\right](i=n, p, e)$ in the extended Thomas-Fermi model with respect to small proton density fluctuations $\delta n_{p}(\mathbf{r})$ around the homogeneous state. The variation of the total energy caused by the inhomogeneity yields

$$
E-E_{0}=\frac{1}{2} \int \frac{d^{3} \mathbf{q}}{(2 \pi)^{3}} v(q)\left|\delta n_{p}(\mathbf{q})\right|^{2},
$$

where $E_{0}$ is the energy of the unperturbed state, $\delta n_{p}(\mathbf{q})$ is the Fourier transform of $\delta n_{p}(\mathbf{r})$, and $v(q)$ is the effective potential between protons given by

$$
v(q)=v_{0}+\beta q^{2}+\frac{4 \pi e^{2}}{q^{2}+\kappa_{e}^{2}} .
$$

The first term in the right side of Eq. (45) is the bulk contribution, and the second is the gradient contribution (for detailed expressions for $v_{0}$ and $\beta$, see Ref. [12]). The potential $v(q)$ takes a minimum value $v_{\min }$ at $q=Q$, where

$$
\begin{aligned}
Q^{2} & =\left(\frac{4 \pi e^{2}}{\beta}\right)^{1 / 2}-\kappa_{e}^{2}, \\
v_{\min } & =v_{0}+2\left(4 \pi e^{2} \beta\right)^{1 / 2}-\beta \kappa_{e}^{2} .
\end{aligned}
$$

The condition for the proton clustering instability, $v_{\min } \leq$ 0 , is satisfied at densities below a critical density $n_{\text {inst }}$. This is because the bulk contribution $v_{0}$, which is dominant in $v_{\text {min }}$, is an increasing function of $n_{\mathrm{b}}$ at densities around $v_{0}, v_{\min } \sim 0$ (see, e.g., Fig. 2 in Ref. [18]). In the presence of the electron screening the critical density $n_{\text {inst }}$ becomes higher since the Coulomb interaction between charge inhomogeneities, which tends to suppress the proton clustering, is weakened by the electron screening through the term $-\beta \kappa_{e}{ }^{2}(<0)$ in Eq. (47).

In summary we have examined the electron screening in the inhomogeneous phases of nuclear matter and clarified its influence on the zero-temperature phase diagram of neutron star matter and supernova matter at subnuclear densities. We have found that the density region occupied by the "pasta" phases is slightly expanded by the electron screening in such a way that the three phase boundaries associated with the bubble phases move into a higher density, whereas the other two move into a lower density. This expansion stems from the model independent features that the electron screening makes spherical nuclei more subject to fission by enlarging the equilibrium size through modifications of the size equilibrium condition as in Eq. (40) and that the role played by the Coulomb interaction between small charge inhomogeneities in uniform nuclear matter in suppressing the proton clustering instability is weakened by the electron screening.

Finally, we consider on what physical conditions a mixed state composed of two phases having different charge density appear as in the nuclear liquid-gas mixed state studied here. As mentioned in Ref. [19] in the context of a quark-hadron mixed state, a mixed state can be stable only when the charge screening and surface tension are sufficiently weak. In the present nuclear 
case, the charge screening is weak and mainly reduces the electrostatic energy, leading to a larger density range of the mixed state for typical values of the surface tension. In the case of the quark-hadron mixed state, the charge screening can be strong enough to raise the bulk energy of the system and hence prefer the phase separated state over the mixed state for realistic values of the surface tension, as discussed in Ref. [20].

\section{Acknowledgments}

The authors are grateful to T. Tatsumi for insightful discussions and comments. G.W. is also grateful to
K. Sato, K. Yasuoka, and T. Ebisuzaki for continuous encouragement. This work was supported in part by RIKEN Junior Research Associates Grant No. J130026, by a Grant-in-Aid for Scientific Research provided by the Ministry of Education, Culture, Sports, Science, and Technology of Japan through Grant No. 14-7939, and by RIKEN Special Postdoctoral Researchers Grant No. 01152040 .
[1] C. J. Pethick and D. G. Ravenhall, Annu. Rev. Nucl. Part. Sci. 45, 429 (1995).

[2] D. G. Ravenhall, C. J. Pethick, and J. R. Wilson, Phys. Rev. Lett. 50, 2066 (1983).

[3] M. Hashimoto, H. Seki, and M. Yamada, Prog. Theor. Phys. 71, 320 (1984).

[4] C.P. Lorenz, D.G. Ravenhall, and C.J. Pethick, Phys. Rev. Lett. 70, 379 (1993).

[5] K. Oyamatsu, Nucl. Phy. A561, 431 (1993).

[6] R. D. Williams and S. E. Koonin, Nucl. Phys. A435, 844 (1985).

[7] M. Lassauat, H. Flocard, P. Bonche, P.H. Heenen, and E. Suraud, Astron. Astrophys. 183, L3 (1987).

[8] G. Watanabe, K. Iida, and K. Sato, Nucl. Phys. A676, 455 (2000); Erratum, Nucl. Phys. A (in press).

[9] G. Watanabe, K. Iida, and K. Sato, Nucl. Phys. A687, 512 (2001); Erratum, Nucl. Phys. A (in press).

[10] F. J. Dyson, Ann. Phys. 63, 1 (1971).

[11] G. Watanabe, K. Sato, K. Yasuoka, and T. Ebisuzaki, Phys. Rev. C 66, 012801(R) (2002); Phys. Rev. C (in press), nucl-th/0308007

[12] G. Baym, H. A. Bethe, and C. J. Pethick, Nucl. Phys. A175, 225 (1971).

[13] D. Z. Freedman, Phys. Rev. D 9, 1389 (1974).

[14] K. Sato, Prog. Theor. Phys. 53, 595 (1975); Prog. Theor. Phys. 54, 1325 (1975).

[15] K. Oyamatsu and M. Yamada, Nucl. Phys. A578, 181 (1994).

[16] P. Magierski and P.-H. Heenen, Phys. Rev. C 65, 045804 (2002).

[17] D.G. Ravenhall, C.D. Bennett, and C.J. Pethick, Phys. Rev. Lett. 28, 978 (1972).

[18] K. Iida, G. Watanabe, and K. Sato, Prog. Theor. Phys. 106, 551 (2001).

[19] H. Heiselberg, C.J. Pethick, and E.F. Staubo Phys. Rev. Lett. 70, 1355 (1993).

[20] D. N. Voskresensky, M. Yasuhira, and T. Tatsumi, Phys. Lett. B541, 93 (2002); Nucl. Phys. A723, 291 (2003). 